\title{
Closed-form Multicast Precoding for Satellite Flexible Payloads
}

\author{
Miguel Ángel Vázquez ${ }^{\dagger}$, Xavier Artiga ${ }^{\dagger}$, Ana I. Pérez-Neira *† \\ ${ }^{*}$ Dept. Signal Theory and Communications - Universitat Politecnica de Catalunya (UPC), Barcelona, Spain, \\ ${ }^{\dagger}$ Centre Tecnologic de Telecomunicacions de Catalunya (CTTC/CERCA), Castelldefels, Barcelona, Spain \\ emails: $\{$ mavazquez, xartiga, aperez $\} @$ cttc.es
}

\begin{abstract}
This paper investigates a novel closed-form noniterative precoding technique for multicast multibeam satellite systems. Next-generation satellite systems will be benefited from the flexible use of the satellite resources especially its power flexible allocation among beams. Intending to obtain a lowcomputational complexity design, we revisit the well-known signal-to-leakage-and-noise ratio design for multicast transmission. Two alternatives are introduced considering both the physical meaning of the ratio and certain multicast channel vector mapping. We observe the benefits of these techniques in satellite flexible payloads. The proposed technique shows a substantial gain compared to the benchmark according to the numerical simulations. Intuitive insights on the precoding behaviour are also presented.
\end{abstract}

Index Terms-Multibeam satellite systems, precoding, closedform beamforming.

\section{INTRODUCTION}

While the feasibility analysis of the application of multiuser multiple-input-multiple-output (MU-MIMO) techniques in multibeam satellite systems was initiated almost 20 years ago [1], it has not been until these days where the first proofsof-concept have been set up [2], [3]. The motivation of these advances has been always the efficient use of the satellite forward link (user segment Space-to-Earth link) spectrum which is normally separated into chunks to avoid inter-beam interference. The mentioned demonstrations are relevant milestones in the satellite communication field, although the path towards including multibeam satellite precoding in ground segment products is still unclear.

Curiously, research and development of satellite flexible payloads have been promoted in parallel to multibeam precoding techniques. Despite original monolithic payloads where both power and frequency allocation among beams is quasifixed for the satellite life, 2021 will see the launch of satellite systems that can be adapted to different user rate demands. This is the case of Eutelsat Quantum [4].

Flexible payload radio-frequency equipment allows reconsidering certain design criteria of multibeam precoding designs. Indeed, payloads for broadband applications are generally constructed with array fed reflectors. In this context, precoding computed at the ground segment shall consider perfeed element power constraint. On the contrary, flexible pay-

This work is funded by Ministry of Science, Innovation and Universities, Spain, under project TERESA -TEC2017-90093-C3-1-R (AEI/FEDER, UE) and by Catalan government under the grant 2017-SGR-01479 loads will consist of direct radiating elements whose available power can be shared among beams. This leads to a substantial change of the original per-feed constraints towards the sumpower constraint.

Multibeam precoding techniques have been guided by the legacy digital video broadcasting second-generation X (DVBS2X) [5] standard. This standard defines the notion of a SuperFrame (SF) which is simply an air-interface framework for synchronising the transmission of different beams. One of its modes is coined as Bundled Frames which imposes the same channel coding and modulation to the whole SF. Due to scheduling latency limitations of the system, Bundled Frames are filled with useful information from different user terminals, leading to the so-called multigroup-multicast transmission ${ }^{1}$.

Considering that the precoding matrix shall be computed in a few milliseconds, the authors have focused on closedform low complexity alternatives for dealing with the precoded multigroup-multicast transmission. This is the case of the seminal paper of G. Taricco, [6] which revisits the regularized zero-forcer or minimum mean square error (MMSE) precoding design [7] considering the multigroup-multicast transmission under per-feed power constraints. The precoding design is boiled down into finding a good mapping between the different channel vectors of users to be served under the same Bundled Frame into a single vector. This multicast mapping is a key element for converting consolidated closed-form precoding unicast designs into multi-group multicast ones.

In this paper, we continue the investigation of one-shot precoding designs with multicast mapping. In particular, we investigate the signal-to-leakage-and-noise ratio (SLNR) [8] beamforming design and its application to multigroup-multicast transmission. We revisit the different terms of desired signal power and interference leakage for a multicast communication scenario and we update its design. The resulting precoding techniques coined as generalized SLNR (G-SLNR) yields larger data rates compared to other alternatives yet preserving a very reduced computational complexity. This is shown in a variety of scenarios. Intuition insights behind the mapping and design are also presented to support the novel approach.

\footnotetext{
${ }^{1}$ DVB-S2X air interface support for multibeam precoding techniques is still under investigation by both academia and industry. Recent DVB-S2X SF modes may be more convenient than the current Bundle Frames approach. This is out of the scope of the current paper and it is left for further works.
} 
The rest of the paper is organized as follows. Section II introduces the system model. Section III presents the novel precoding design and certain insights regarding its capabilities. Section IV shows the numerical results and Section V concludes.

\section{System Model And Problem Statement}

Let us consider the satellite transmission of $K$ independent symbols, $s_{k} \quad k=1, \ldots, K$. The satellite flexible payload is equipped with a direct-radiating array (DRA) of $N$ antennas whose available power can be completely shared among beams. All symbols are statistically independent and they have unitary energy. User terminals are grouped in clusters (beams) and we denote them by $\mathcal{B}_{k}$ for $k=1, \ldots, K$. Each group is assumed to have an arbitrary number of users $\left|\mathcal{B}_{k}\right|$. A total number of users $L$ is considered.

Assuming full frequency reuse among beams, the satellite performs precoding in order to mitigate interference. In this context, the received signal by the $i$-th user at the $k$-th group can be written as

$$
y_{k, i}=\mathbf{h}_{k, i}^{H}\left(\sum_{k=1}^{K} \mathbf{w}_{k} s_{k}\right)+n_{k, i}
$$

where $\mathbf{h}_{k, i} \in \mathbb{C}^{N \times 1}$ is the equivalent channel vector between the satellite gateway and the $i$-th user at the $k$-th group, $\mathbf{w}_{k}$ for $k=1, \ldots, K$ are the precoding vectors. Transmitted symbols are denoted by $s_{k}$ and they are zero mean with variance equal to $\sigma^{2}$. The additive white Gaussian noise term is denoted by $n_{k, i}$ assumed to have unit variance and zero mean.

Clear sky channel conditions are assumed and the channel vectors are modelled such that

$$
\mathbf{h}_{k, i}=\frac{G_{R}}{4 \pi \frac{d_{k}}{\lambda}} \mathbf{\Phi} \mathbf{e}_{k, i}
$$

$d_{k, i}$ is the distance between the $i$-th and the $k$-th group and the satellite. $\lambda$ is the carrier wavelength. Vector $\mathbf{e}_{k, i}$ is the steering vector between the satellite DRA and the $i$-th user at the $k$ th beam. Matrix $\boldsymbol{\Phi}_{k, i} \in \mathbb{C}^{N \times N}$ is a diagonal matrix whose elements model the channel phase effects. These effects are described by the $n$-th diagonal entry

$$
\psi_{n}=\theta_{k, i}^{\mathrm{RF}}+\theta_{k, i}^{\mathrm{LNB}},
$$

where $\theta_{k, i}^{\mathrm{RF}}=\frac{2 \pi}{\lambda} d_{k, i}$ is the phase rotation due to the radiofrequency signal propagation which depends on the user distance to the satellite, $\theta_{k, i}^{\mathrm{LNB}}$ is the phase contribution of the receiver low noise block downconverters assumed to be Gaussian with zero mean and standard deviation of 0.24 degrees.

We assume the use of Bundled Frames where information from multiple users is encapsulated with the same modulation and error protecting codes. This results in the following attainable rates per group

$$
R_{k}=\min _{i \in \mathcal{B}_{k}} \log _{2}\left(1+\frac{\left|\mathbf{h}_{k, i}^{H} \mathbf{w}_{k}\right|^{2}}{\sum_{j \neq k}^{K}\left|\mathbf{h}_{k, i}^{H} \mathbf{w}_{j}\right|^{2}+\sigma^{2}}\right),
$$

where the multicast precoding $\mathbf{w}_{j}, j=1, \ldots, K$ must be designed to diminish the interference as we explain in the present work. Note that the term multicast in this context is an abuse of language. Indeed, users belonging to the same group will receive its own dedicated information but due to air-interface and upper layers specifications, these information chunks have to be encapsulated using the same modulation and channel coding.

For the sake of comparison, this paper also considers as a benchmark a satellite payload that, despite it has full flexibility in terms of spatial processing, it considers a pre-fixed beam allocation. Similarly to [9] for array fed reflector payloads, we consider a pre-processing unitary matrix $\mathbf{B} \in \mathbb{C}^{N \times K}$ that creates a pre-fixed beampattern.

In this context, the channel vectors are updated as follows

$$
\mathbf{H}^{\text {fixed beams }}=\mathbf{H B} \text {, }
$$

where

$$
\mathbf{H}=\left(\mathbf{H}_{1}^{T}, \ldots, \mathbf{H}_{K}^{T}\right)^{T},
$$

being

$$
\mathbf{H}_{k}=\left(\mathbf{h}_{k, 1}^{T}, \ldots, \mathbf{h}_{k,\left|\mathcal{B}_{k}\right|}^{T}\right)^{T}
$$

As a matter of fact, this pre-processing limits the spatial resolution of the on-ground precoding techniques while it reduces the feeder link bandwidth requirements. Indeed, this pre-fixed beams approach requires $B K$ feeder link bandwidth while the flexible payload needs $B N$, being $N \gg K$.

In the following we consider the flexible payload approach, all derivations apply when we consider $\mathbf{H}^{\text {fixed beam }}$ instead of H.

\section{PRECODING FORMULATION}

\section{A. SLNR beamforming technique}

In this paper we revisit the known approach of SLNR [8] for the multibeam satellite scenario. This technique, which is originally conceived for unicast transmission (e.g. $\left|\mathcal{B}_{k}\right|=1$ ), consists of the optimization for each beamforming vector $k$ of

$$
\frac{\mathbf{w}_{k}^{\mathrm{H}} \mathbf{h}_{k} \mathbf{h}_{k}^{\mathrm{H}} \mathbf{w}_{k}}{\sum_{j \neq k}^{K} \mathbf{w}_{k}^{\mathrm{H}} \mathbf{h}_{j} \mathbf{h}_{j}^{\mathrm{H}} \mathbf{w}_{k}+\sigma^{2}}
$$

where we have omitted the additional sub-index in $\mathbf{h}_{k}$ as we are considering a unicast transmission. The notion of this ratio is motivated by the authors in [8] as the aim of the beamforming vector is to have a large radiated power towards the intended user, $\mathbf{w}_{k}^{\mathrm{H}} \mathbf{h}_{k} \mathbf{h}_{k}^{\mathrm{H}} \mathbf{w}_{k}$, this value has to be large compared to the noise power at the receiver $\sigma^{2}$ and the radiated power towards the rest of the users, $\sum_{j \neq k}^{K} \mathbf{w}_{k}^{\mathrm{H}} \mathbf{h}_{j} \mathbf{h}_{j}^{\mathrm{H}} \mathbf{w}_{k}$. Remarkably, this ratio with certain updates can be also interpreted as beamforming radiofrequency directivity as presented in [10].

Given a maximum power allocation per beamforming vector such that

$$
\left\|\mathbf{w}_{k}\right\|^{2} \leq \frac{P}{K}
$$


the beamforming design that optimizes (8) reads

$$
\mathbf{w}_{k}^{\mathrm{SLNR}}=\sqrt{\frac{P}{K}} \text { max-geigen }\left(\mathbf{h}_{k} \mathbf{h}_{k}^{\mathrm{H}}, \sum_{j \neq k}^{K} \mathbf{h}_{j} \mathbf{h}_{j}^{\mathrm{H}}+\frac{K \sigma^{2}}{P} \mathbf{I}\right),
$$

Mimicking the SLNR approach we shall now upgrade the notion on noise experienced by the receiver. However, we do not longer have a single receiver, but multiple in each group. In this context, we propose the following ratio to optimize

$$
\frac{\left\|\mathbf{H}_{k} \mathbf{w}_{k}\right\|^{2}}{\sum_{j \neq k}^{K}\left\|\mathbf{H}_{j} \mathbf{w}_{k}\right\|^{2}+\frac{L}{K} \sigma^{2}},
$$

where the numerator considers the radiated power towards all the intended users in a group. In the denominator, $\sum_{j \neq k}^{K}\left\|\mathbf{H}_{j} \mathbf{w}_{k}\right\|^{2}$ is the power of the leakage towards all the non-intended users. Finally, as the physical meaning of $\sigma^{2}$ is blurred in the definition applied to multicast systems, we opt to consider a scaled version of the noise power considering the ratio $\frac{L}{K}$. Assuming that the cardinality of the user groups is equally distributed, the virtual total noise power becomes $\frac{L}{K} \sigma^{2}$.

We coin this last ratio in (11) as generalized SLNR (GSLNR), because it is able to tackle multicast transmissions. Note that this definition differs to the original approach reported in [8] for multiuser MIMO systems. It is easy to observe that the beamforming vector that optimizes (11) is

$\mathbf{w}_{k}^{\mathrm{G}-\mathrm{SLNR}}=\sqrt{\frac{P}{K}}$ max-geigen $\left(\mathbf{H}_{k}^{\mathrm{H}} \mathbf{H}_{k}, \sum_{j \neq k}^{K} \mathbf{H}_{j}^{\mathrm{H}} \mathbf{H}_{j}+\frac{L \sigma^{2}}{P} \mathbf{I}\right)$.

\section{B. Average Mapping}

Despite the presented G-SLNR captures the communications idea of ratio between intended transmit power and unintended transmitted power, we know that multibeam satellite systems have a peculiar behaviour.

The consolidated literature on multibeam satellite system [11] shows that the MMSE with channel averaging yields to the most adequate complexity-performance trade-off. Due to this, there might of interest to re-consider our proposed GSLNR with this idea.

The use of channel averaging was initially formulated by Taricco in [6]. The main motivation behind this idea is to try to re-use a known closed-form precoding technique (i.e. MMSE) for multicast transmission. Mathematically, the problem boils down to find a mapping

$$
f\left(\mathbf{H}_{k}\right) \rightarrow \mathbf{g}_{k}
$$

so that vector $\mathbf{g}_{k} \in \mathbb{C}^{N \times 1}$ captures the spatial signature of all users within the same group. A simple yet efficient technique is to consider the average

$$
\mathbf{g}_{k}^{\mathrm{av}}=\frac{1}{\left|\mathcal{B}_{k}\right|} \sum_{i=1}^{\left|\mathcal{B}_{k}\right|} \mathbf{h}_{k, i}
$$

This later technique is reconsidered in [12] via a smart average considering the channel phase effect

$$
\mathbf{g}_{k}^{\text {art }}=\frac{1}{\left|\mathcal{B}_{k}\right|} \sum_{i=1}^{\left|\mathcal{B}_{k}\right|} e^{j \chi_{k, i}} \mathbf{h}_{k, i}
$$

where

$$
\chi_{k, i}=\angle\left[\mathbf{h}_{k, i}\right]_{l},
$$

where

$$
l=\arg \max _{n=1, \ldots, N}\left|\left[\mathbf{h}_{k, i}\right]_{n}\right| .
$$

In other words, we rotate the channel vector phases using the phase of the entry with the highest channel gain. The objective is to cancel the effects of $\theta_{k, i}^{\mathrm{RF}}$ and $\theta_{k, i}^{\mathrm{LNB}}$ in the channel average. These phase components are constant across the channel vectors, but they are different from channel vector to channel vector. Therefore, after averaging, the resulting channel vector shows phase variation across their components due to the addition of components with different $\theta_{k, i}^{\mathrm{RF}}$ and $\theta_{k, i}^{\mathrm{LNB}}$. The ultimate effect is that the pointing accuracy of the averaged channel is randomized depending on those channel phase components, as will be shown in the next section, which yields to the performance loss already reported in [12].

In light of the promising results reported in [12] we opt to adapt our proposed G-SLNR with this averaging mapping. With this aim, we can re-write our original proposal in (11) such that

$$
\frac{\mathbf{w}_{k}^{\mathrm{H}} \mathbf{g}_{k}^{\text {art }} \mathbf{g}_{k}^{\mathrm{H}, \text { art }} \mathbf{w}_{k}}{\sum_{j \neq k}^{K} \mathbf{w}_{k}^{\mathrm{H}} \mathbf{g}_{j}^{\text {art }} \mathbf{g}_{j}^{\mathrm{H}, \text { art }} \mathbf{w}_{k}+\frac{L}{K} \sigma^{2}} .
$$

It is easy to observe that in contrast to the original G-SLNR option, consider the average mapping offers us the possibility of avoiding the eigenvector computation and the expression becomes

$$
\mathbf{w}_{k}^{\mathrm{G}-S L N R-a r t}=\sqrt{\frac{P}{K}}\left(\sum_{j \neq k}^{K} \mathbf{g}_{k}^{\text {art }} \mathbf{g}_{k}^{\text {art }, H}+\frac{L}{P} \sigma^{2}\right)^{-1} \mathbf{g}_{k}^{\text {art }} .
$$

Remarkably, this precoding design has the same computational complexity as the well-known multicast MMSE [11]

$$
\mathbf{W}^{\mathrm{MMSE}}=\gamma_{\mathrm{MMSE}}\left(\mathbf{G}^{H} \mathbf{G}+\frac{K}{P} \mathbf{I}\right)^{-1} \mathbf{G}
$$

being $\mathbf{G}$ the channel matrix collapsing the user channel averages of all groups and $\gamma_{\text {MMSE }}$ a normalizing factor for restricting the total transmit power below $P$.

\section{Performance Insights}

Inferring the performance behaviour of the introduced precoding designs to the multibeam satellite capacity exceeds the scope of the current paper. Instead, we opt to provide certain ad-hoc analysis on how the precoding, and more specifically, its pointing capability, behaves on a particular scenario. This discussion flavours the numerical analysis performed in the next Section.

Figure 1 shows the array factor of a uniform linear array with 20 elements spaced $\lambda / 2$ considering: (i) the matched filter 


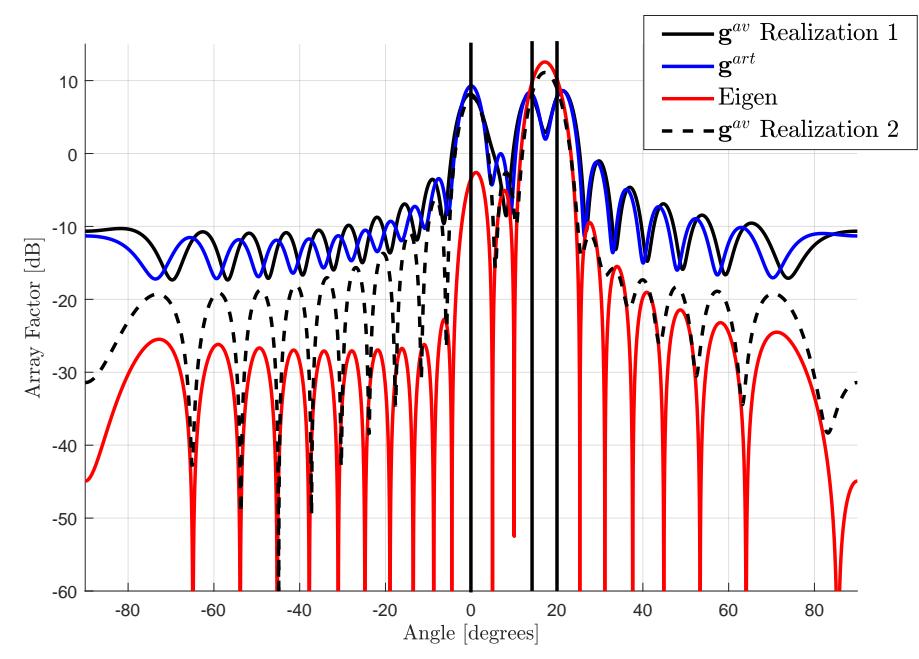

Fig. 1. Array gain factor of $\mathbf{g}^{\text {art }}, \mathbf{g}^{\text {av }}$ for two realizations and the eigenvector associated with the maximum eigenvalue of $\mathbf{H}_{k}^{H} \mathbf{H}_{k}$.

to $\mathbf{g}_{k}^{\text {art }}$; (ii) the matched filter to the eigenvector associated to the maximum eigenvalue of $\mathbf{H}_{k}^{H} \mathbf{H}_{k}$; (iii) and two realizations of the matched filter to $\mathbf{g}_{k}^{\mathrm{av}}$, considering different $\theta_{k, i}^{\mathrm{RF}}$ and $\theta_{k, i}^{\mathrm{LNB}}$ phase components. Three intended user at $\left[0^{\circ}, 15^{\circ}, 20^{\circ}\right]$ are considered.

It can be observed that the average mapping with phase rotation synthesises a multibeam pattern pointing almost equally to the three users, whereas the eigenvector does not equally treat all intended users. The behaviour of the average mapping without phase rotation depends on the specific aforementioned phase components, which randomize the pointing capabilities of this solution.

As it can be observed, the solution based on the maximum eigenvector shows a clear array gain imbalance between the different intended users. This is not the case of $\mathrm{g}^{\text {art }}$ where all users obtain similar array gain. This feature makes the average mapping adequate for the multicast transmission as all users should have similar array gain.

\section{NUMERICAL RESULtS}

We consider MEO satellite located at $\left(13^{\circ},-173^{\circ}\right)$ and at a height of $8000 \mathrm{Km}$ providing service to randomly distributed users across the Caribbean area. The satellite is equipped with a square DRA of $6 \times 6$ circular aperture antennas (i.e. horn like) with diameter and spacing of $1.6 \lambda$, thus trading off the number of elements and the grating lobes created outside the coverage area. The DRA can be used as a full flexible payload, or it can be operated to produce fixed beams as depicted in Figure 2.

As a main key performance indicator of the system we consider the sum-rate defined as

$$
\mathcal{S R}=B \sum_{k=1}^{K} R_{k},
$$

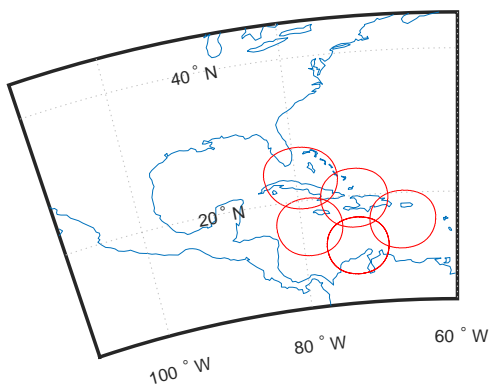

Fig. 2. Fixed beams coverage area over the Caribbean sea.

TABLE I

SATELLITE System SPECIFICATIONS

\begin{tabular}{c|c} 
Maximum radiated power $(P)$ & $1120 \mathrm{~W}$ \\
Channel bandwidth $(B)$ & $500 \mathrm{MHz}$ \\
Carrier frequency & $20 \mathrm{GHz}$ \\
User terminal G/T & $17.94 \mathrm{~dB}$ \\
DRA element gain & $14 \mathrm{~dB}$
\end{tabular}

where $B$ is the available bandwidth considered $500 \mathrm{MHz}$. We assume a pure line-of-sight system whose parameters are defined in Table 1.

We evaluate two different scenarios. In the one hand, Figure 3 shows the sum-rate versus the total number of users considering a random clustering. In other words, we assume that the scheduler does not take into account the proximity of user terminals for allocating them in the same SF. This of course degrades the performance of precoding. On the other hand, we consider that scheduling is performed based on a $k$-means clustering in Figure 4. In particular, we consider a number of clusters equal to $K$ for which we calculate the resulting precoding matrix and sum-rate.

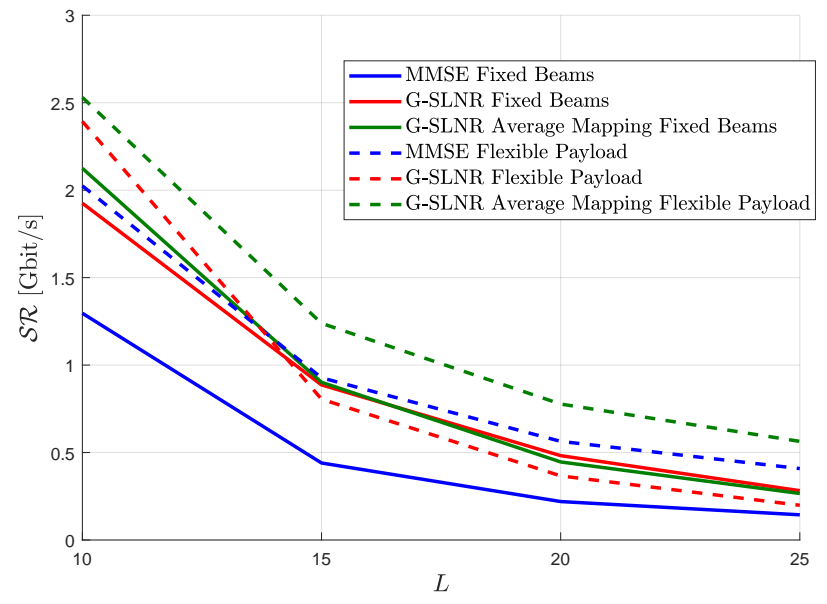

Fig. 3. Sum-rate evaluation versus the number of user terminals $L$ with both a flexible payload and a payload performing fixed beams. Random clustering is employed for user grouping.

In both cases we compare a full flexible payload scenario 


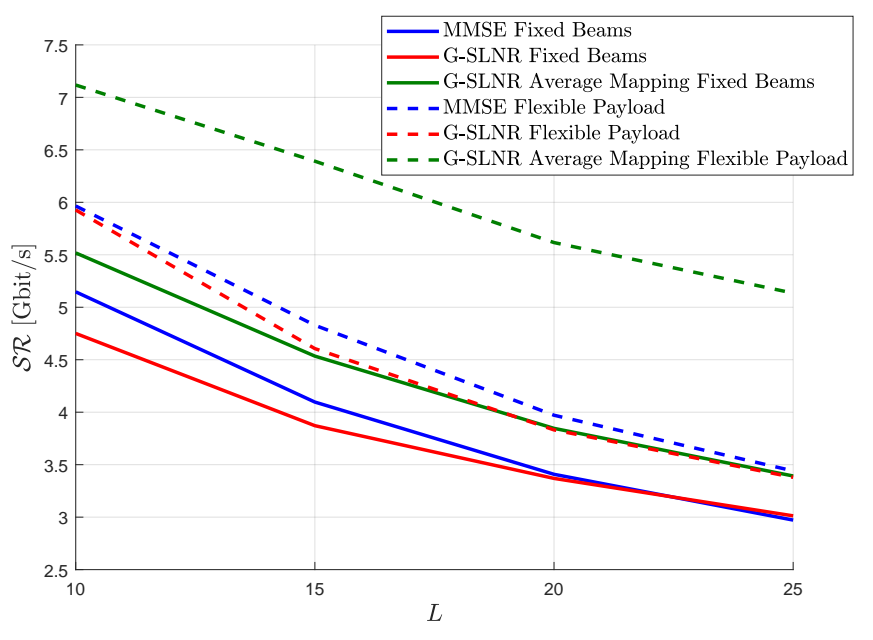

Fig. 4. Sum-rate evaluation versus the number of user terminals $L$ with both a flexible payload and a payload performing fixed beams. It is considered k-means clustering for the user grouping.

with the alternative based on a payload equipped with fixed beams. As it can be observed, larger attainable rates can be obtained when full flexible payload capabilities are employed. Among all options, G-SLNR with average mapping results into the best option. This alternative provides a substantial gain for the case with $k$-means user grouping, while for random grouping the sum-rate gain is reduced.

It is important to remark that our proposed scheme shows larger sum-rate values compared to the benchmark MMSE scheme while offering the same computational complexity.

\section{CONCLUSiOns}

This paper presented a novel precoding design coined GSLNR that showed a better performance compared to the benchmark in terms of sum-rate. The proposed design preserves a low-complexity and; thus, it is adequate for deployments. The design was derived from the original and wellknown unicast SLNR design and two different adaptations to the multicast case were introduced. The one using a clever user channel vector averaging was the one that yielded the largest sum-rate. This was shown in a close-to-real scenario considering a flexible payload with DRAs.

\section{REFERENCES}

[1] G. Caire, L. Cottatelluci, M. Debbah, G. Lechner, and R. Muller, "Interference mitigation techniques for satellite systems," TR1/TR2 Report of ESTEC Contract, no. 18070/04, 2004.

[2] K. Storek, R. T. Schwarz, and A. Knopp, "Multi-Satellite Multi-User MIMO Precoding: Testbed and Field Trial," in ICC 2020 - 2020 IEEE International Conference on Communications (ICC), 2020, pp. 1-7.

[3] J. C. Merlano Duncan, J. Querol Borras, N. Maturo, J. Krivochiza, D. Spano, S. Norshahida, L. Martinez Marrero, S. Chatzinotas, and B. Ottersten, "Hardware Precoding Demonstration in Multi-Beam UHTS Communications under Realistic Payload Characteristics," in Proceedings of the 37th International Communications Satellite Systems Conference, 2019.

[4] "Eutelsat quantum," https://www.eutelsat.com/en/satellites/futurelaunches.html?eutelsat-quantum, accessed: 16-02-2021.
[5] E. ETSI, "Digital video broadcasting (dvb); second generation framing structure, channel coding and modulation systems for broadcasting, interactive services, news gathering and other broadband satellite applications," Part II: S2-Extensions (DVB-S2X), pp. 22-27, 2005.

[6] G. Taricco, "Linear Precoding Methods for Multi-Beam Broadband Satellite Systems," in European Wireless 2014; 20th European Wireless Conference, 2014, pp. 1-6.

[7] C. B. Peel, B. M. Hochwald, and A. L. Swindlehurst, "A vector-perturbation technique for near-capacity multiantenna multiuser communication-part I: channel inversion and regularization," IEEE Transactions on Communications, vol. 53, no. 1, pp. 195-202, 2005.

[8] M. Sadek, A. Tarighat, and A. H. Sayed, "A Leakage-Based Precoding Scheme for Downlink Multi-User MIMO Channels," IEEE Transactions on Wireless Communications, vol. 6, no. 5, pp. 1711-1721, 2007.

[9] V. Joroughi, M. Vázquez, A. I. Pérez-Neira, and B. Devillers, "Onboard Beam Generation for Multibeam Satellite Systems," IEEE Transactions on Wireless Communications, vol. 16, no. 6, pp. 3714-3726, 2017.

[10] M. Lagunas, A. Perez-Neira, and X. Artiga, "Array factor directivity for interference scenarios," in Proceedings of the 19th International Conference on Systems, 2015, pp. 105-111.

[11] A. I. Perez-Neira, M. A. Vazquez, M. R. B. Shankar, S. Maleki, and S. Chatzinotas, "Signal processing for high-throughput satellites: Challenges in new interference-limited scenarios," IEEE Signal Processing Magazine, vol. 36, no. 4, pp. 112-131, 2019.

[12] X. Artiga and M. Á. Vázquez, "Effects of Channel Phase in Multibeam Multicast Satellite Precoding Systems," in Proceedings of 37th International Communications Satellite Systems Conference (ICSSC), 28-1 November, 2019, Okinawa (Japan), Nov. 2019. 